\title{
Bilateral advanced (group D or E) intraocular retinoblastoma: outcomes in 72 Asian Indian patients
}

\author{
Swathi Kaliki ${ }^{1} \cdot$ Priya Mittal $^{1,2} \cdot$ Sashwanthi Mohan ${ }^{1,2} \cdot$ Goura Chattannavar $^{1,2} \cdot$ Sai Divya Jajapuram ${ }^{1} \cdot$ \\ Ashik Mohamed $\mathbb{D}^{3} \cdot$ Vijay Anand Reddy Palkonda ${ }^{1}$
}

Received: 9 November 2018 / Revised: 28 January 2019 / Accepted: 6 February 2019 / Published online: 1 April 2019

(c) The Royal College of Ophthalmologists 2019

\begin{abstract}
Purpose To study the clinical presentation, treatment, and outcomes of patients with bilateral advanced intraocular retinoblastoma.

Methods Retrospective case series of 72 patients.

Results The mean age at presentation was 19 months. Leukocoria $(n=49,68 \%)$ was the most common presenting complaint. The tumors were classified as groups $\mathrm{D}(n=60,42 \%)$ or $\mathrm{E}(n=84,58 \%)$ based on the Philadelphia version of International Classification of Retinoblastoma (ICRB); groups D $(n=84,58 \%)$ or $\mathrm{E}(n=60,42 \%)$ based on Children's Hospital Los Angeles version of International Classification of Intraocular Retinoblastoma (ICIoR); T2 $(n=116,81 \%)$ or T3 $(n=28,19 \%)$ based on 8th edition American Joint Committee Classification (AJCC). Systemic chemotherapy $(n=138$, 96\%) was the most common primary treatment modality. The chance of globe salvage was higher for group D based on ICRB (83\%; odds ratio (OR) 7.73; 95\% confidence interval (CI) $3.45-17.33$ ) or ICIoR $(81 \%$; OR 12.75 ; 95\% CI $5.74-$ 28.34) and T2b (73\%; OR 5.19; 95\% CI 2.51-10.73) based on AJCC. Over a mean follow-up period of 59 months, tumor recurrence was noted in $42(29 \%)$ eyes and globe salvage was achieved in $83(58 \%)$ eyes. Of the 50 eyes where vision was recorded, vision of 20/200 or better was achieved in $24(48 \%)$ eyes. There were events of leukemia $(n=1,1 \%)$, pinealoblastoma $(n=1,1 \%)$, systemic metastasis $(n=3,4 \%)$, and death $(n=4,6 \%)$ during the follow-up period.

Conclusion Multimodality treatment allows globe salvage (58\%) and vision salvage (48\%) in eyes with advanced group D and $\mathrm{E}$ intraocular retinoblastoma.
\end{abstract}

\section{Introduction}

In the recent times, globe salvage rates for retinoblastoma (RB) have drastically improved with the expanded armamentarium for the management of RB. However, the globe salvage rates for advanced intraocular $\mathrm{RB}$ remain

Supplementary information The online version of this article (https:// doi.org/10.1038/s41433-019-0409-z) contains supplementary material, which is available to authorized users.

Swathi Kaliki

kalikiswathi@yahoo.com

1 Operation Eyesight Universal Institute for Eye Cancer, Hyderabad, India

2 Academy for Eye Care Education, Hyderabad, India

3 Ophthalmic Biophysics Laboratory, L V Prasad Eye Institute, Hyderabad, India suboptimal. In the developing countries, late presentation of retinoblastoma is not uncommon, resulting in high rates of enucleation. In the developed nations, globe salvage rates are better due to early presentation of $\mathrm{RB}$ and timely intervention.

Various treatment protocols including bilateral enucleation, bilateral external beam radiotherapy, systemic chemotherapy, enucleation of severely affected eye and external beam radiotherapy for the less advanced eye, combination of systemic chemotherapy and external beam radiotherapy, combination of systemic chemotherapy and plaque radiotherapy with or without focal treatment, simultaneous intraarterial chemotherapy (IAC) with or without intravitreal chemotherapy have been used in the management of bilateral RB [1-13]. The globe salvage rates with these various forms of treatment ranges from 0 to $91 \%$ [1-13]. In eyes with bilateral advanced intraocular retinoblastoma (bilateral Reese-Ellsworth IV or V), the globe salvage rates were $30-61 \%$ [4, 6, 8]. In this study, we 
discuss our experience with globe salvage following the treatment of bilateral advanced intraocular retinoblastoma in Asian Indian patients.

\section{Methods}

This is a retrospective interventional case series. Institutional review board approval was obtained for the study. The study adhered to the tenets of the Declaration of Helsinki. All cases of RB treated at the Operation Eyesight Universal Institute for Eye Cancer, L V Prasad Eye Institute between the years January 2000 and March 2015 were reviewed. Advanced intraocular RB was defined as tumor classified as group D or E based on Philadelphia version of International Classification of Retinoblastoma (ICRB) [14] or Children's Hospital Los Angeles (CHLA) version of International Classification of Intraocular Retinoblastoma (ICIoR) [15]; and T2 or T3 tumors based on 8th edition American Joint Committee Classification (AJCC) [16]. Those patients with bilateral advanced intraocular RB with a minimum period of 6 months follow-up after completion of primary treatment were included in the study. Those patients with tumors belonging to groups $\mathrm{A}, \mathrm{B}, \mathrm{C}$ based on ICRB or ICIOR, or T1 and T4 based on AJCC, or those with orbital extension of RB in either eye were excluded from the study. The patients with incomplete data or inadequate follow-up were also excluded from the study.

The demographic data and clinical history including age, gender, family history of $\mathrm{RB}$, and presenting complaints were recorded. The anterior and posterior segment details were recorded. The presence or absence of orbital pseudocellulitis, megalocornea, secondary glaucoma, iris neovascularization, and ectropion uveae were noted. Based on the fundus findings, the tumors were classified according to ICRB, ICIoR, and AJCC. All patients underwent orbital imaging (computed tomography or magnetic resonance imaging) to rule out optic nerve extension or extraocular tumor extension. Bone marrow biopsy and cerebrospinal fluid aspiration was done in all cases with group D3 or E tumor based on ICRB. The details of primary treatment (systemic chemotherapy, enucleation) were recorded. Systemic chemotherapy protocol included 6-9 cycles of threeweekly high-dose vincristine, etoposide, and carboplatin. If optic nerve transection involvement or microscopic scleral or extrascleral tumor extension was noted on histopathology post-enucleation, a total of 12 cycles of high-dose chemotherapy and orbital external beam radiotherapy were administered as per protocol. Primary enucleation was offered to all patients with cT3 tumor. Adjuvant treatment was defined as additional treatment given during the primary treatment and thereafter till complete regression of the tumor. Secondary treatment was defined as initiation of treatment for tumor recurrence after complete regression of the tumor. The details of adjuvant or secondary treatment (cryotherapy, transpupillary thermotherapy, periocular chemotherapy, intravitreal chemotherapy, intraarterial chemotherapy, systemic chemotherapy, enucleation, plaque radiotherapy, external beam radiotherapy) were also noted. Repeat systemic chemotherapy protocol included 6-9 cycles of three-weekly high-dose vincristine, etoposide, and carboplatin. Few patients needed multiple repetitions of the chemotherapy protocol in view of multiple recurrences. When applicable, the reason for secondary treatment was recorded.

Tumor recurrence was defined as any new tumor in the form of subretinal seeds, vitreous seeds, or solid tumor after complete regression of tumor following treatment. The events of tumor recurrence, enucleation, second cancer, systemic metastasis, and death were recorded. The treatment details of recurrent tumor were also noted. When applicable, histopathology data were recorded. Vision testing was performed in children using Snellens visual acuity chart. It was recorded in children following complete tumor control and in those who needed no further examination under anesthesia. All children were beyond 4 years of age at the time of visual acuity recording.

\section{Statistical analysis}

The statistical analysis was performed using the software Origin v7.0 (OriginLab Corporation, Northampton, MA, USA). Categorical data were described by proportions and compared between the groups by Chi-square test or Fisher's exact test. Continuous data such as age at presentation and duration of follow-up were checked for normality by Shapiro-Wilk test. Since the distribution was not normal, they were described in median and interquartile range and compared between the groups by Kruskal-Wallis test. In case of multiple pairwise comparisons, Bonferroni correction was applied. A $p$-value of less than 0.05 was considered statistically significant. Kaplan-Meier (KM) survival analysis was performed to estimate the probability of globe salvage. Cox proportional hazards regression analysis was done to compare the survival probabilities between the various groups in the classification of retinoblastoma.

\section{Results}

Of the 1592 RB cases examined during the study period, $134(8 \%)$ patients had bilateral advanced intraocular RB. Of these, 25 patients had less than 6-months follow-up, and 37 patients had inadequate data due to primary treatment 
Table 1 Bilateral advanced (group D or E) intraocular retinoblastoma: demographics and clinical presentation

\begin{tabular}{lc}
\hline Feature & $n(\%)$ \\
& $n=72$ \\
\hline Age at presentation (months) & \\
Mean (median, range) & $19(12,3-96)$ \\
Gender & \\
$\quad$ Male & $38(53)$ \\
Female & $34(47)$ \\
Family history of retinoblastoma & $11(15)$ \\
Presenting complaints* & \\
$\quad$ Leukocoria & $49(68)$ \\
Strabismus & $6(8)$ \\
Red eye & $5(7)$ \\
Enlarged eye & $4(6)$ \\
Decreased vision & $13(18)$ \\
Nystagmus & $1(1)$ \\
Symptoms & \\
Asymptomatic & $0(0)$ \\
Complaints in only one eye & $38(53)$ \\
Complaints in both eyes & $34(47)$ \\
\hline
\end{tabular}

$* n>72$ since few patients had more than one presenting complaint

elsewhere before presenting to us and were thus excluded from the study. Seventy-two patients with bilateral advanced intraocular RB with a minimum follow-up of 6 months post treatment were included in the study.

The demographic and clinical details are listed in Table 1. The mean age at presentation with bilateral advanced intraocular RB was 19 months (median, 12 months; range, 3-96 months). There were 38 (53\%) males and $34(47 \%)$ females. Leukocoria $(n=49,68 \%)$ and decreased vision $(n=13,18 \%)$ were the common presenting complaints. Presenting complaints were noted in only one eye $(n=38,53 \%)$ or in both eyes $(n=34,47 \%)$. Iris neovascularization $(n=23,16 \%)$ and secondary glaucoma $(n=21,15 \%)$ were the common anterior segment signs. Fourteen $(10 \%)$ patients had both features of iris neovascularization and secondary glaucoma. The tumors were classified as group $\mathrm{D}(n=60,42 \%)$ or $\mathrm{E}(n=84,58 \%)$ based on ICRB; group $\mathrm{D}(n=84,58 \%)$ or $\mathrm{E}(n=60,42 \%)$ based on ICIoR; T2a $(n=28,19 \%)$, T2b $(n=88,61 \%)$, or T3 $(n=28,19 \%)$ based on AJCC (Table 2). Based on tumor classifications, the combination of groups $\mathrm{D}$ and $\mathrm{E}$ in each eye ( $25 \%$ based on ICRB; $28 \%$ based on ICIoR) was more common, and bilateral T2 (31\%) was more common based on AJCC.

The treatment details are listed in Table 3. Though primary enucleation was offered to all patients with cT3 tumor, only six patients with cT3c underwent primary enucleation based on acceptance of procedure by the parents. The remaining
Table 2 Bilateral advanced (group D or E) intraocular retinoblastoma: tumor features and classification

\begin{tabular}{|c|c|}
\hline Feature & $\begin{array}{l}n(\%) \\
n=144\end{array}$ \\
\hline Orbital pseudocellulitis & $3(2)$ \\
\hline Megalocornea & $13(9)$ \\
\hline Secondary glaucoma & $21(15)$ \\
\hline Pseudohypopyon & $3(2)$ \\
\hline Hyphema & $5(3)$ \\
\hline Iris neovascularization & $23(16)$ \\
\hline Ectropion uveae & $17(12)$ \\
\hline \multicolumn{2}{|c|}{$\begin{array}{l}\text { International Classification of Retinoblastoma } \\
\text { (Philadelphia version) }\end{array}$} \\
\hline Group D & $60(42)$ \\
\hline Group E & $84(58)$ \\
\hline Bilateral group D & $12(8)$ \\
\hline Bilateral group E & $24(17)$ \\
\hline Combination of groups D and E & $36(25)$ \\
\hline \multicolumn{2}{|c|}{$\begin{array}{l}\text { International Classification of Retinoblastoma (Children's } \\
\text { Hospital Los Angeles version) }\end{array}$} \\
\hline Group D & $84(58)$ \\
\hline Group E & $60(42)$ \\
\hline Bilateral group D & $22(15)$ \\
\hline Bilateral group E & $10(7)$ \\
\hline Combination of groups $\mathrm{D}$ and $\mathrm{E}$ & $40(28)$ \\
\hline \multicolumn{2}{|l|}{ 8th edition AJCC } \\
\hline $\mathrm{T} 2 \mathrm{a}$ & $28(19)$ \\
\hline $\mathrm{T} 2 \mathrm{~b}$ & $88(61)$ \\
\hline $\mathrm{T} 3 \mathrm{a}$ & $3(2)$ \\
\hline $\mathrm{T} 3 \mathrm{~b}$ & $1(<1)$ \\
\hline $\mathrm{T} 3 \mathrm{c}$ & $17(12)$ \\
\hline $\mathrm{T} 3 \mathrm{~d}$ & $4(3)$ \\
\hline $\mathrm{T} 3 \mathrm{e}$ & $3(2)$ \\
\hline Bilateral T2 & $45(31)$ \\
\hline Bilateral T3 & $1(<1)$ \\
\hline Combination of $\mathrm{T} 2$ and $\mathrm{T} 3$ & $26(18)$ \\
\hline
\end{tabular}

AJCC American Joint Committee Classification

cases underwent enucleation during the course of systemic chemotherapy. Systemic chemotherapy $(n=138,96 \%)$ was the most common primary treatment modality. Adjuvant treatment was needed for $84(58 \%)$ eyes during or after primary treatment. Transpupillary thermotherapy $(n=66,46 \%)$ was the most common adjuvant treatment modality. Of the six eyes that underwent primary enucleation, two eyes had highrisk features, including ciliary body invasion $(n=1)$ and massive choroidal invasion $(n=1)$. Both these patients received adjuvant systemic chemotherapy. Secondary treatment was needed in $42(29 \%)$ eyes with tumor recurrence. The most common secondary treatment was transpupillary thermotherapy $(n=27,19 \%)$. Eleven eyes $(8 \%)$ needed 
Table 3 Bilateral advanced (group D or E) intraocular retinoblastoma: treatment and outcomes

\begin{tabular}{|c|c|}
\hline Feature & $\begin{array}{l}n(\%) \\
n=144\end{array}$ \\
\hline \multicolumn{2}{|l|}{ Primary treatment } \\
\hline Systemic chemotherapy & $138(96)$ \\
\hline Enucleation & $6(4)$ \\
\hline \multicolumn{2}{|l|}{ Adjuvant treatment* } \\
\hline Transpupillary thermotherapy & $66(46)$ \\
\hline Cryotherapy & 24 (17) \\
\hline Periocular carboplatin & 19 (13) \\
\hline Systemic chemotherapy & $2(1)$ \\
\hline External beam radiotherapy & $14(10)$ \\
\hline Secondary enucleation & $45(33)$ \\
\hline \multicolumn{2}{|l|}{ Secondary treatment ${ }^{*}$} \\
\hline Transpupillary thermotherapy & 27 (19) \\
\hline Cryotherapy & $18(13)$ \\
\hline Periocular carboplatin & $5(3)$ \\
\hline Plaque radiotherapy & $3(2)$ \\
\hline Intraarterial chemotherapy & $4(3)$ \\
\hline Systemic chemotherapy & 19 (13) \\
\hline External beam radiotherapy & $1(1)$ \\
\hline Secondary enucleation & $11(8)$ \\
\hline Total number of chemotherapy cycles Mean (median, range) & $9(6,6-30)$ \\
\hline Tumor recurrence & $42(29)$ \\
\hline \multicolumn{2}{|l|}{ Globe salvage } \\
\hline Both eyes enucleated & $12(17)$ \\
\hline Both eyes salvaged & $23(32)$ \\
\hline One eye salvaged & $37(51)$ \\
\hline \multicolumn{2}{|l|}{ Visual acuity ( $n=50$ eyes) } \\
\hline$>20 / 200$ & $24(48)$ \\
\hline$<20 / 200$ & $26(52)$ \\
\hline \multicolumn{2}{|l|}{ Second cancer } \\
\hline Leukemia & $1(1)$ \\
\hline Pinealoblastoma & $1(1)$ \\
\hline Systemic metastasis & $3(4)$ \\
\hline Death & $4(6)$ \\
\hline
\end{tabular}

*Few patients needed more than one form of treatment secondary enucleation for tumor recurrence. Nineteen (13\%) patients needed further systemic chemotherapy. Overall, the mean number of chemotherapy cycles for complete tumor regression was 9 (median, 6, range, 6-30). Ten patients needed more than 12 cycles of chemo to combat multiple tumor recurrences and achieve complete tumor control. During the follow-up period, secondary enucleation was done in $56(41 \%)$ eyes owing to poor response to systemic chemotherapy $(n=40,71 \%)$, tumor recurrence $(n=11,20 \%)$, non-visualization of tumor due to vitreous hemorrhage $(n=3$, $5 \%)$, or cataract $(n=2,4 \%)$. Of these 56 enucleated eyes, 11 $(20 \%)$ eyes harbored high-risk histopathology features, including iris invasion $(n=3)$, ciliary body invasion $(n=3)$, massive choroidal invasion $(n=7)$, postlaminar optic nerve invasion $(n=2)$, scleral invasion $(n=2)$, and microscopic extrascleral invasion $(n=2)$. Nine patients were lost to follow-up during the course of treatment and reviewed back with worsening of the globe status.

Over a mean follow-up period of 59 months (median, 46 months; range, 6-189 months), bilateral globe salvage was achieved in $23(32 \%)$, unilateral globe salvage was achieved in 37 (51\%), and both eyes were enucleated in 12 $(17 \%)$ cases (Table 3 ). Of those patients who underwent bilateral enucleation, $83 \%$ patients had bilateral group E eyes based on ICRB, $50 \%$ group E eyes based on ICIoR, while based on AJCC, combination of T2 and T3 (58\%) 
Table 4 Globe salvage: analysis based on tumor classification

\begin{tabular}{lcclc}
\hline Feature & $\begin{array}{l}\text { Globe } \\
\text { salvaged } \\
n=83\end{array}$ & $\begin{array}{c}\text { Enucleation } \\
n(\%)\end{array}$ & $\begin{array}{l}n \text {-value } \\
n(\%)\end{array}$ & $\begin{array}{l}\text { Odds ratio } \\
\text { (95\% confidence } \\
\text { interval) }\end{array}$ \\
\hline International Classification of & & & & \\
Retinoblastoma (Philadelphia version) & & & & \\
Group D & $50(60)$ & $10(16)$ & $<0.0001$ & $7.73(3.45-17.33)$ \\
Group E & $33(40)$ & $51(84)$ & $<0.0001$ & $0.13(0.06-0.29)$ \\
International Classification of & & & & \\
Retinoblastoma (Children's Hospital & & & & \\
Los Angeles) & $68(82)$ & $16(26)$ & $<0.0001$ & $12.75(5.74-28.34)$ \\
Group D & $15(18)$ & $45(74)$ & $<0.0001$ & $0.08(0.04-0.17)$ \\
Group E & & & & \\
8th edition AJCC & $19(23)$ & $9(15)$ & 0.32 & - \\
T2a & $64(77)$ & $24(39)$ & $<0.0001$ & $5.19(2.51-10.73)$ \\
T2b & $0(0)$ & $3(5)$ & 0.07 & - \\
T3a & $0(0)$ & $1(2)$ & 0.42 & - \\
T3b & $0(0)$ & $17(28)$ & $<0.0001$ & na \\
T3c & $0(0)$ & $4(7)$ & 0.03 & - \\
T3d & $0(0)$ & $3(5)$ & 0.07 & - \\
T3e & & & &
\end{tabular}

na not applicable since one value is 0 was common. The chance of globe salvage was higher for group D based on ICRB (50 (83\%) of 60 group D globes salvaged; odds ratio (OR) $7.73 ; 95 \%$ confidence interval (CI) 3.45-17.33) or ICIoR (68 (81\%) of 84 group D globes salvaged; OR 12.75; 95\% CI 5.74-28.34) and T2b (64 (73\%) of 88 globes salvaged; OR 5.19; 95\% CI 2.51-10.73) based on AJCC (Table 4).

The KM estimates of globe salvage are listed in Table 5 (Supplementary Fig. 1). KM estimate of globe salvage of group D vs. group E eyes based on ICRB was $95 \%$ vs. 52\% at 1 year, $82 \%$ vs. $36 \%$ at 5 years, and $67 \%$ vs. $30 \%$ at 10 years $(p<0.0001$, hazards ratio $(\mathrm{HR})=5.14)$ (Supplementary Fig. 2). KM estimate of globe salvage of group D vs. group E eyes based on ICIoR was $94 \%$ vs. $36 \%$ at 1 year, $78 \%$ vs. $21 \%$ at 5 years, and $62 \%$ vs. $21 \%$ at 10 years $(p<$ $0.0001, \mathrm{HR}=8.26$ ) (Supplementary Fig. 3). KM estimate of globe salvage of T2 vs. T3 eyes based on AJCC was $83 \%$ vs. $15 \%$ at 1 year, $69 \%$ vs. $0 \%$ at 5 years, and $56 \%$ vs. $0 \%$ at 10 years $(p<0.0001, \mathrm{HR}=11.87)$ (Supplementary Fig. 4). Of the 83 eyes saved, Snellen's visual acuity was recorded in 50 eyes. Of these 50 eyes with recorded visual acuity, vision $>20 / 200$ was achieved in $24(48 \%)$ eyes, of which vision $>$ 20/40 was evident in $16(32 \%)$ eyes. Strabismus was noted in $28(56 \%)$ eyes and nystagmus in $9(18 \%)$ eyes.

There were events of leukemia $(n=1,1 \%)$, pinealoblastoma $(n=1,1 \%)$, systemic metastasis $(n=3,4 \%)$, and death $(n=4,6 \%)$ during the follow-up period. All three patients with systemic metastasis and the patient with pinealoblastoma died during the follow-up period. Of these four cases, two patients had bilateral group E and two had a combination of groups D and E based on ICRB; all four had a combination of groups D and E based on ICIoR; three patients had bilateral T2 and one had a combination of T2 and T3 at presentation. All four patients who eventually died were started on primary intravenous chemotherapy. Two patients were non-compliant to treatment and were lost to follow-up during the course of treatment and reviewed back with advanced disease, including intracranial tumor extension one year later in one case, and extraocular tumor extension 7 months later in the second case.

\section{Discussion}

Bilateral involvement is noted in $25-40 \%$ cases with retinoblastoma. The treatment of bilateral retinoblastoma has evolved from bilateral enucleation to globe salvage treatments [1-13]. Bilateral external beam radiotherapy as a primary treatment for bilateral RB became less popular overtime due to increased risk of second non-ocular cancers and subsequent mortality [17]. Systemic chemotherapy with or without radiation (low dose EBRT or plaque radiotherapy) or focal treatment (cryotherapy or laser therapy) has shown promising results in bilateral RB, achieving globe salvage in 64-92\% eyes [6-8, 10]. In our study of bilateral advanced intraocular RB, systemic chemotherapy was the most common primary modality $(n=138$ eyes, 96\%). Over a mean follow-up period of 59 months, globe 
Table 5 Globe salvage: KaplanMeier survival analysis based on tumor classification

\begin{tabular}{|c|c|c|c|c|c|}
\hline \multirow{2}{*}{$\begin{array}{l}\text { Feature } \\
\text { Time point }\end{array}$} & \multicolumn{4}{|c|}{ Probability of globe salvage } & \multirow[t]{2}{*}{$p$-value } \\
\hline & Year 1 & Year 3 & Year 5 & Year 10 & \\
\hline Overall & $70 \% \pm 4 \%$ & $60 \% \pm 4 \%$ & $55 \% \pm 5 \%$ & $44 \% \pm 7 \%$ & \\
\hline \multicolumn{6}{|c|}{ International Classification of Retinoblastoma (Philadelphia version) } \\
\hline Group D & $95 \% \pm 3 \%$ & $89 \% \pm 4 \%$ & $82 \% \pm 6 \%$ & $67 \% \pm 11 \%$ & \multirow[t]{2}{*}{$<0.0001$} \\
\hline Group E & $52 \% \pm 6 \%$ & $41 \% \pm 6 \%$ & $36 \% \pm 6 \%$ & $30 \% \pm 7 \%$ & \\
\hline \multicolumn{6}{|c|}{ International Classification of Retinoblastoma (Children's Hospital Los Angeles version) } \\
\hline Group D & $94 \% \pm 3 \%$ & $88 \% \pm 4 \%$ & $78 \% \pm 6 \%$ & $62 \% \pm 10 \%$ & \multirow[t]{2}{*}{$<0.0001$} \\
\hline Group E & $36 \% \pm 6 \%$ & $21 \% \pm 6 \%$ & $21 \% \pm 6 \%$ & $21 \% \pm 6 \%$ & \\
\hline \multicolumn{6}{|c|}{ 8th edition AJCC } \\
\hline $\mathrm{T} 2$ & $83 \% \pm 4 \%$ & $74 \% \pm 4 \%$ & $69 \% \pm 5 \%$ & $56 \% \pm 9 \%$ & \multirow{3}{*}{0.59} \\
\hline $\mathrm{T} 2 \mathrm{a}$ & $74 \% \pm 9 \%$ & $69 \% \pm 9 \%$ & $63 \% \pm 10 \%$ & $63 \% \pm 10 \%$ & \\
\hline $\mathrm{T} 2 \mathrm{~b}$ & $86 \% \pm 4 \%$ & $76 \% \pm 5 \%$ & $71 \% \pm 6 \%$ & $46 \% \pm 15 \%$ & \\
\hline $\mathrm{T} 3$ & $15 \% \pm 7 \%$ & $4 \% \pm 4 \%$ & $0 \%$ & $0 \%$ & $<0.0001^{\mathrm{c}}$ \\
\hline
\end{tabular}

AJCC American Joint Cancer Committee

${ }^{a}$ Hazards ratio: 5.14; ${ }^{b}$ hazards ratio: 8.26; ${ }^{c}$ hazards ratio: 11.87 salvage was achieved in $83(58 \%)$ eyes. Additional EBRT was needed for $15(10 \%)$ eyes.

Recently, simultaneous IAC in both eyes has shown good results with high rates of globe salvage and minimal systemic complications. In a review of 60 patients with bilateral RB treated with simultaneous IAC, 116 eyes were salvaged with KM estimate of ocular survival at $99 \%$ at 1 year, $97 \%$ at 3 years, and $95 \%$ at 5 years [12]. In another study from the same group, the 3-year KM estimate of ocular survival was $91 \%$ with the use of IAC and intravitreal chemotherapy [13]. In our study, IAC was not used as a primary treatment in any case, but was used as a salvage treatment in four eyes. Of these four eyes, two eyes were salvaged while two eyes underwent enucleation due to poor response to treatment.

Globe salvage in RB depends on severity of disease at presentation. The classification of RB differs based on the classification system. Reese-Ellsworth Classification system was very popular till 2000's when it was replaced with International Classification of Retinoblastoma. Philadelphia version ICRB [14] and CHLA version of ICIoR [15] are similar but differ in the classification of advanced disease, groups D and E. While the subset of tumor filling $>50 \%$ of the globe is considered as group E by ICRB, it may be classified as group D based on ICIoR, if the tumor is not touching the lens $[14,15]$. Furthermore, to provide uniformity in tumor classification and thus facilitate outcome analysis for multicenter trials, AJCC was proposed. Currently AJCC is in its 8th edition [16]. Based on ICRB, systemic chemotherapy and focal treatment can salvage $47 \%$ group D eyes [14], while IAC and intravitreal chemotherapy have increased the globe salvage rate of group D eyes to $94 \%$, and $36 \%$ of group E eyes can also be salvaged [18]. Based on Children's Oncology Group classification of RB, which is similar to ICIoR [15] classification of advanced $\mathrm{RB}$, the globe salvage rate of group $\mathrm{D}$ eyes is $92 \%$ and group $\mathrm{E}$ is $71 \%$ in the IAC and intravitreal chemotherapy era [13]. In a study of 64 group D eyes (ICIoR) by Fabian et al., primary intravenous chemotherapy with appropriate adjuvant treatment achieved globe salvage in $63 \%$ cases with no evidence of metastasis or death [19]. In our study from the developing world, where advanced RB is common and systemic chemotherapy is the most commonly used primary treatment modality, based on ICRB, $83 \%$ group D eyes were salvaged and 39\% group E eyes were salvaged. Based on ICIoR, $81 \%$ group D eyes were salvaged and $25 \%$ group E eyes were salvaged. Based on AJCC, 72\% T2 eyes were salvaged while all $\mathrm{T} 3$ eyes were enucleated owing to very advanced disease at presentation. In our series, with the use of primary intravenous chemotherapy and appropriate adjuvant treatment, the globe salvage rate of group D eyes (ICRB and ICIoR) was better at $81-83 \%$ compared with other published series at $47-63 \%$. This could be related to referral bias and the differences in the size of retinal tumor and extent of subretinal and vitreous seeding.

Intravenous chemotherapy may offer systemic protective effect against development of systemic metastasis and pinealoblastoma [20]. The rates of pinealoblastoma and systemic metastasis can be higher in patients with advanced intraocular RB that are treated with IAC without systemic chemotherapy. Thus IAC should be used with caution in cases with advanced intraocular RB. In our series, three cases developed systemic metastasis and one patient developed pinealoblastoma during the course of disease, in spite of receiving systemic intravenous chemotherapy. Eventually, all four patients died due to the disease.

The risk-benefit ratio between enucleation versus globe salvage treatment for eyes with advanced intraocular RB 
should be carefully assessed since these eyes have a chance of harboring high-risk RB, which can potentially be fatal. In a comparison of 524 patients with RB from India and United States of America, it was noted that Asian Indians had a fivefold greater risk of having optic nerve invasion and threefold greater risk of massive choroidal invasion compared with Americans [21]. The incidence of high-risk $\mathrm{RB}$ in group D eyes is $15-17 \%$ and in group E eyes is 24 $50 \%$ [22-24]. RB eyes with clinical features predictive of high-risk RB are best managed by enucleation rather than globe salvage treatment strategies. Globe salvage treatment in eyes with advanced retinoblastoma is also associated with more number of examinations under anesthesia (EUA) (average number of EUA's, 16) compared with those undergoing primary enucleation (average number of EUA's, 7), which may affect the neurological and cognitive development in a child [25].

The ultimate goals of successful RB treatment are life salvage with prevention of metastasis, globe salvage, vision salvage, and improved quality of life. In eyes with advanced intraocular RB (groups D and E), vision salvage is a challenge due to diffuse retinal involvement and obscuration of macula. In a study of 32 group D ((ICIoR) RB eyes salvaged with multimodal management, $50 \%$ eyes had $<20$ / 200 vision, $60 \%$ had strabismus, and $22 \%$ had nystagmus [26]. In our study, vision could be recorded in 50 of 83 salvaged eyes, and of these, $52 \%$ eyes had $<20 / 200$ vision, $56 \%$ had strabismus, and $18 \%$ eyes had nystagmus.

The drawback of our study is the retrospective nature of the study. In conclusion, all patients who had cT3 had primary or secondary enucleation. All patients who had primary enucleation survived over a mean follow-up period of 5 years. Three of 22 patients (14\%) who had an initial attempt at globe salvage died despite secondary enucleation. Our current protocol is to provide high-dose systemic chemotherapy (Vincristine, Etoposide, and Carboplatin) to patients presenting with bilateral advanced intraocular retinoblastoma. Primary enucleation is done in eyes with very advanced tumor (AJCC T3 tumor) on presentation followed by systemic chemotherapy for the second eye. IAC is used as a salvage treatment in these eyes when systemic chemotherapy fails. With these treatment strategies, globe salvage (58\%) and vision salvage (48\%) can be achieved even in cases with bilateral advanced intraocular retinoblastoma.

\section{Summary}

\section{What was known before}

- Globe salvage rates of bilateral intraocular retinoblastoma in the White population.

\section{What this study adds}

- Globe salvage rates of bilateral advanced intraocular retinoblastoma from a developing country.

Acknowledgements Support was provided by The Operation Eyesight Universal Institute for Eye Cancer (SK) and Hyderabad Eye Research Foundation (SK), Hyderabad, India. The funders had no role in the preparation, review, or approval of the paper.

\section{Compliance with ethical standards}

Conflict of interest The authors declare that they have no conflict of interest.

Publisher's note: Springer Nature remains neutral with regard to jurisdictional claims in published maps and institutional affiliations.

\section{References}

1. Reese AB, Merriam GR Jr, Martin HE. Treatment of bilateral retinoblastoma by irradiation and surgery; report on 15-year results. Am J Ophthalmol. 1949;32:175-90.

2. Abramson DH, Ronner HJ, Ellsworth RM. Second tumors in nonirradiated bilateral retinoblastoma. Am J Ophthalmol. 1979;87:624-7.

3. Gagnon JD, Ware CM, Moss WT, Stevens KR. Radiation management of bilateral retinoblastoma: the need to preserve vision. Int J Radiat Oncol Biol Phys. 1980;6:669-73.

4. Abramson DH, Ellsworth RM, Tretter P, Adams K, Kitchin FD. Simultaneous bilateral radiation for advanced bilateral retinoblastoma. Arch Ophthalmol. 1981;99:1763-66.

5. Abramson DH, Ellsworth RM, Tretter P, Javitt J, Kitchin FD. Treatment of bilateral groups I through III retinoblastoma with bilateral radiation. Arch Ophthalmol. 1981;99:1761-2.

6. Haye C, Desjardins L, Schlienger P, Zucker JM, Laurent M. Treatment of bilateral retinoblastoma stage $\mathrm{V}$ at the Curie Foundation. 33 cases. Ophthalmic Paediatr Genet. 1987;8:73-76.

7. Gallie BL, Budning A, DeBoer G, Thiessen JJ, Koren G, Verjee Z, et al. Chemotherapy with focal therapy can cure intraocular retinoblastoma without radiotherapy. Arch Ophthalmol. 1996;114:1321-28.

8. Kingston JE, Hungerford JL, Madreperla SA, Plowman PN. Results of combined chemotherapy and radiotherapy for advanced intraocular retinoblastoma. Arch Ophthalmol. 1996;114:1339-43.

9. Lee V, Hungerford JL, Bunce C, Ahmed F, Kingston JE, Plowman PN. Globe conserving treatment of the only eye in bilateral retinoblastoma. Br J Ophthalmol. 2003;87:1374-80.

10. Sohajda Z, Damjanovich J, Bárdi E, Szegedi I, Berta A, Kiss C. Combined local treatment and chemotherapy in the management of bilateral retinoblastomas in Hungary. J Pediatr Hematol Oncol. 2006;28:399-401.

11. Berry JL, Jubran R, Kim JW, Wong K, Bababeygy SR, Almarzouki $\mathrm{H}$, et al. Long-term outcomes of Group $\mathrm{D}$ eyes in bilateral retinoblastoma patients treated with chemoreduction and low-dose IMRT salvage. Pediatr Blood Cancer. 2013;60:688-93.

12. Abramson DH, Marr BP, Francis JH, Dunkel IJ, Fabius AW, Brodie SE, et al. Simultaneous Bilateral ophthalmic artery chemosurgery for bilateral retinoblastoma (Tandem therapy). PLoS ONE. 2016;11:e0156806.

13. Francis JH, Roosipu N, Levin AM, Brodie SE, Dunkel IJ, Gobin YP, et al. Current treatment of bilateral retinoblastoma: the impact 
of intraarterial and intravitreous chemotherapy. Neoplasia. 2018;20:757-63.

14. Shields CL, Mashayekhi A, Au AK, Czyz C, Leahey A, Meadows $\mathrm{AT}$, et al. The International Classification of Retinoblastoma predicts chemoreduction success. Ophthalmology. 2006;113: 2276-80.

15. Linn Murphree A. Intraocular retinoblastoma: the case for a new group classification. Ophthalmol Clin North Am. 2005;18:41-53.

16. Mallipatna AC, Gallie BL, Chévez-Barrios P, Rouic LL, Chantada GL, Doz F, et al. Retinoblastoma. In: Amin MB, Edge SB, Greene FL, et al., editors. AJCC Cancer Staging Manual. 8th edition. Switzerland: Springer; 2017. p. 819-31.

17. Abramson DH, Ellsworth RM, Zimmerman LE. Nonocular cancer in retinoblastoma survivors. Trans Sect Ophthalmol Am Acad Ophthalmol Otolaryngol. 1976;81(3 Pt 1):454-7.

18. Shields CL, Manjandavida FP, Lally SE, Pieretti G, Arepalli SA, Caywood $\mathrm{EH}$, et al. Intra-arterial chemotherapy for retinoblastoma in 70 eyes: outcomes based on the international classification of retinoblastoma. Ophthalmology. 2014;121:1453-60.

19. Fabian ID, Stacey AW, Johnson KP, Onadim Z, Chowdhury T, Duncan C, et al. Primary intravenous chemotherapy for group D retinoblastoma: a 13-year retrospective analysis. Br J Ophthalmol. 2017;101:82-88.

20. Ramasubramanian A, Kytasty C, Meadows AT, Shields JA, Leahey A, Shields CL. Incidence of pineal gland cyst and pineoblastoma in children with retinoblastoma during the chemoreduction era. Am J Ophthalmol. 2013;156:825-9.

21. Kaliki S, Shields CL, Eagle RC Jr, Iram S, Shields JA. High-risk intraocular retinoblastoma: Comparison between Asian Indians and Americans from two major referral centers. Retina. 2018;38:2023-9.

22. Kaliki S, Shields CL, Rojanaporn D, Al-Dahmash S, McLaughlin JP, Shields JA, et al. High-risk retinoblastoma based on international classification of retinoblastoma: analysis of 519 enucleated eyes. Ophthalmology. 2013;120:997-1003.

23. Wilson MW, Qaddoumi I, Billups C, Haik BG, RodriguezGalindo C. A clinicopathological correlation of 67 eyes primarily enucleated for advanced intraocular retinoblastoma. $\mathrm{Br} \mathrm{J}$ Ophthalmol. 2011;95:553-8.

24. Kaliki S, Srinivasan V, Gupta A, Mishra DK, Naik MN. Clinical features predictive of high-risk retinoblastoma in 403 Asian Indian patients: a case-control study. Ophthalmology. 2015;122:1165-72.

25. Fabian ID, Stacey AW, Johnson KC, Chowdhury T, Duncan C, Reddy MA, et al. Primary enucleation for group D retinoblastoma in the era of systemic and targeted chemotherapy: the price of retaining an eye. Br J Ophthalmol. 2018;102:265-71.

26. Fabian ID, Naeem Z, Stacey AW, Chowdhury T, Duncan C, Reddy MA, et al. Long-term visual acuity, strabismus, and nystagmus outcomes following multimodality treatment in group D retinoblastoma eyes. Am J Ophthalmol. 2017;179:137-44. 